\title{
Estudio comparativo de la distribución espacial del muérdago enano (Arceuthobium sp.) en la ladera norte del Parque Nacional Nevado de Toluca, México
}

\author{
A comparative study of the spatial distribution of the dwarf mistletoe (Arceuthobium sp.) \\ on the north slope of the National Park Nevado de Toluca, Mexico
}

\author{
José Francisco Ramírez Dávilaa*, Elvia Porcayo Camargo ${ }^{\mathrm{b}}$ \\ *Autor de correspondencia: a Universidad Autónoma del Estado de México UAEM, Facultad de Ciencias Agrícolas, CP 50200, \\ Toluca, México, tel.: 01 729- 2965531 ext: 139, jfrd@uaemex.mx \\ b Universidad Autónoma del Estado de México UAEM, Facultad de Geografía, 50200, Toluca, México.
}

\begin{abstract}
SUMMARY
This paper shows how, by applying geostatistical methods, maps of incidence of a parasite called coniferous dwarf mistletoe (Arceuthobium sp.) can be obtained; and thereof the spatial distribution, in the northern slope of the National Park Nevado de Toluca, is possible to be known. That distribution was achieved by establishing both methods: Statistical Not Space and Space and Statistics (Geostatistics). The results showed that populations of dwarf mistletoe in the region studied showed a distribution aggregate, with several centers of aggregation. Prepared density maps of dwarf mistletoe technique, using Krige, corroborated the same kind of distribution mentioned. We found that this parasite infestation of conifers does not reach $100 \%$ of the study plots; an interesting situation to establish control measures targeted to areas of present infestation. To determine a spatial and temporal stability of the populations of the parasite was not achieved. The information gathered is useful for the understanding of spatial behavior and pest control, whose impact on the region studied has significantly increased. A comparison with the results to determine the spatial distribution of the parasite was_performed through Statistics Not Space.
\end{abstract}

Key words: geostatistics, semivariograms, Krige, spatial modeling, dwarf mistletoe.

\section{RESUMEN}

En este trabajo se demuestra mediante la aplicación de métodos geoestadísticos que se pueden obtener mapas de incidencia de un hemiparásito de coníferas llamado muérdago enano (Arceuthobium sp.), y así conocer la distribución espacial de éste, en la ladera norte del Parque Nacional Nevado de Toluca. Los resultados mostraron que las poblaciones de muérdago enano de la región estudiada tienen una distribución agregada, con varios centros de agregación. Se elaboraron mapas de densidad de la plaga mediante la técnica del krigeado, los cuales corroboraron el tipo de distribución mencionado. Se halló que la infestación del hemiparásito no alcanzó la totalidad de las parcelas estudiadas, lo cual resulta interesante para establecer estrategias y medidas de control específicas en las zonas de infestación. La información recabada es de gran utilidad para el conocimiento del comportamiento espacial y control de esta plaga, cuya incidencia en la región estudiada ha aumentado considerablemente. Se realizó además, una comparación de los resultados de distribución espacial del hemiparásito obtenidos mediante estadística espacial y no espacial.

Palabras clave: geoestadística, semivariogramas, krigeado, modelización espacial, muérdago enano.

\section{INTRODUCCIÓN}

La Secretaría de Ecología (1999) señala que el Parque Nacional Nevado de Toluca (PNNT) es una región montañosa que se ubica en la parte central del Estado de México, al Sur-Oeste del Valle de Toluca entre los $18^{\circ} 59^{\prime}$ y $19^{\circ} 13^{\prime}$ de latitud norte y los $99^{\circ} 33^{\prime}$ y $99^{\circ} 58^{\prime}$ longitud oeste. Según PROTIMBOS (1972), tiene un total de 48.571 ha, de las cuales el 77,42\% tiene uso forestal y de éste más del $50 \%$ corresponde a bosques con poca densidad, combinados con usos agrícolas y ganaderos.

El Nevado de Toluca o Xinantécatl, según CEPANAF (1979), es un volcán extinto que alcanza una altura de
$4.558 \mathrm{~m}$ s.n.m. El Parque tiene una abundante vegetación de Pinus montezuma Lamb., Pinus hartwegii Lindl., Abies religiosa Kunth Schltdl. et Cham y Cupressus lusitanica Mill., entre otras, y donde habitan diferentes especies animales. La temperatura media mensual oscila entre $3,0^{\circ} \mathrm{C}$ y $5,8^{\circ} \mathrm{C}$ a los $4.140 \mathrm{~m}$ s.n.m. Las temperaturas más elevadas se registran en mayo con valores superiores a $5^{\circ} \mathrm{C}$ en las áreas más elevadas y de $15^{\circ} \mathrm{C}$ en las estribaciones. Las temperaturas más bajas se registran en enero con un promedio de $3^{\circ} \mathrm{C}$, no obstante las temperaturas extremas decrecen hasta $-15^{\circ} \mathrm{C}$. Las lluvias en esta zona se presentan en verano. Los valores medios oscilan entre los $746 \mathrm{~mm}$ en los sitios cercanos al valle, y los 
$1.243 \mathrm{~mm}$ en la cota de los $4.000 \mathrm{~m}$ s.n.m. y superiores (García 1976).

Algunos de los problemas que presentan las coníferas en el PNNT son la deforestación, los incendios, las enfermedades y las plagas. Entre estas últimas se encuentran las infestaciones del muérdago enano (Arceuthobium sp. Engelm.), el cual se ha convertido en un serio problema sanitario para los bosques de la región mencionada (González 1984). El muérdago enano es una de las plantas hemiparásitas más importantes del grupo de las heterófitas debido a que causa serios perjuicios sobre otros vegetales, al extraer de estos últimos el agua y los nutrientes que necesita (González 1984). La Secretaría de Ecología (1999) indica que en el PNNT las dos especies de pino presentes (Pinus montezumae Lamb. y Pinus hartwegii Lindl.) son atacadas por el muérdago enano.

Los daños que causa el muérdago enano a las coníferas, son: a) hipertrofia del fuste y de las ramas en el punto infestado, b) atrofia y posterior rotura del fuste y de las ramas de las partes infestadas hacia la parte alta debido al peso del hemiparásito, c) deformación de las ramas de los árboles afectados al culminar el ataque, d) notable retardo del crecimiento tanto en altura como en diámetro de los árboles de edad mayor, e) interferencia en la vida normal del árbol, provocando su muerte principalmente si son árboles jóvenes, f) retardo de las funciones de desarrollo, altura y diámetro, especialmente al inicio de la primavera, que es la época de mayor crecimiento de los árboles, g) debilitamiento de los árboles, los que son presa de insectos y otros microorganismos, h) reducción considerable de la producción de semillas fértiles en los árboles atacados, llegando a nulificarlos totalmente. Estos daños son de especial importancia y justifican el establecimiento de programas de control de esta planta hemiparásita (Vega 1976, Verduzco 1976).

De acuerdo con Sawyer (1989) y Hulbert (1990) los métodos comúnmente utilizados en la estadística clásica o no espacial para el estudio de las distribuciones espaciales de los organismos se basan en el uso de las distribuciones estadísticas e índices de dispersión, los cuales no tienen en cuenta la exacta localización espacial de la muestra. Ello produce ciertos efectos indeseables: estos métodos en ocasiones fallan al diferenciar patrones espaciales diferentes y sus descripciones de los patrones espaciales son altamente dependientes del tamaño de las unidades de muestreo y de la relación entre la media y la varianza. A causa de estos problemas y con la disponibilidad de paquetes estadísticos apropiados, se ha incrementado el interés en el uso de la estadística espacial. Una de las ramas de estadística espacial más utilizadas es la geoestadística, que tiene la ventaja de caracterizar la distribución espacial en un espectro de escalas y direcciones múltiples, además de ser independiente de la relación entre la media y la varianza.

El estudio de la distribución espacial del muérdago se ha realizado en otras ocasiones utilizando la estadística clásica o no espacial, lo que ha provocado que la obtención de resultados conlleve mucho tiempo y no existan mapas de localización de la mencionada plaga. Por tal fin, se requieren estudios que permitan determinar la distribución espacial de las poblaciones del hemiparásito con el fin de que se puedan elaborar estrategias de control de esta plaga.

El presente trabajo se basa en el uso de la estadística espacial, particularmente la geoestadística, que se define como la aplicación de la teoría de funciones aleatorias al reconocimiento y estimación de fenómenos naturales (Journel y Huijbregts 1978) o, simplemente, el estudio de las variables numéricas distribuidas en el espacio (Chauvet 1968). Su punto de partida es asumir una intuición topoprobabilista (Matheron 1971). Una definición más actual señala que la geoestadística se define como el estudio estadístico de fenómenos naturales que se distribuyen de forma continua en el espacio o el tiempo (Chilés y Delfiner 1999) y que describe la autocorrelación espacial entre datos muestreados y la usa en varios tipos de modelos espaciales.

Los métodos geoestadísticos proporcionan una medida directa de la dependencia espacial, ya que tienen en cuenta la naturaleza bidimensional de la distribución de los organismos a través de su exacta localización espacial. La geoestadística, además, permite elaborar mapas de gran utilidad de la distribución espacial de un organismo (Isaaks y Srivastava 1988, Oliver y Webster 1991, Rossi et al. 1992, Blom y Fleischer 2001).

El objetivo general de este trabajo consiste en determinar la distribución espacial de las poblaciones de muérdago enano en la ladera norte del PNNT mediante la utilización de la estadística no espacial y de la espacial (análisis geoestadístico) y posteriormente comparar los resultados obtenidos con ambos métodos. La hipótesis de trabajo del estudio supone que la distribución espacial de la planta hemiparásita es agregada.

\section{MÉTODOS}

Para estudiar el comportamiento espacial de las poblaciones de muérdago enano sobre coníferas en la ladera norte del PNNT se establecieron 10 parcelas experimentales de una hectárea de extensión $(100 \times 100 \mathrm{~m})$ cada una de Pinnus montezumae. Las parcelas se establecieron a lo largo de la ladera entre los 3.500 m s.n.m. y los $4.100 \mathrm{~m}$ s.n.m. ya que en este lugar y rango es donde se ha reportado la mayor presencia del muérdago enano (PROTIMBOS 1972).

En cada parcela se estableció una malla rectangular y se muestreó cada 10 metros en ambas direcciones. Cuando se dio el caso de que a los 10 metros no existía una conífera, se buscó un árbol localizado a una distancia máxima de un metro. De este modo, en cada parcela se muestrearon alrededor de 100 árboles. Cada árbol muestreado se georreferenció utilizando un sistema de posicionamiento global diferencial (DGPS) con estación submétrica para 
establecer sus coordenadas espaciales. Se realizó un conteo del número de muérdagos enanos presentes en cada árbol muestreado. Cada árbol fue debidamente etiquetado para conocer su posición exacta. El diámetro a la altura del pecho de los árboles muestreados fue de $69 \mathrm{~cm}$ en promedio, oscilando el mencionado diámetro entre 51 y 83 $\mathrm{cm}$. Los muestreos se realizaron quincenalmente. Cada parcela fue completamente muestreada en dos sesiones. El periodo de muestreos se distribuyó entre mayo y septiembre de 2007.

Se realizaron unos análisis exploratorios estadísticos a los datos originales de las poblaciones de muérdago enano a cada una de las parcelas utilizando el método de curtosis para establecer la normalidad estadística de los datos; cabe señalar que para establecer normalidad los valores del coeficiente de curtosis deben estar en el rango de -3 y 3. También fue necesario realizar una transformación logarítmica de los datos $\left[\log _{10}(n+1)\right]$ para normalizarlos y de este modo poder utilizar sin sesgo alguno los métodos geoestadísticos.

Estadística no espacial. Existen dos métodos generales para establecer la distribución espacial de los organismos: las distribuciones estadísticas (binomial negativa y Poisson) y los índices de dispersión (de dispersión y de Green); ambos se utilizaron en este estudio para realizar la comparación pertinente entre lo hallado con la estadística no espacial y la estadística espacial. Para todas las distribuciones estadísticas se utilizó el programa MLP de máxima verosimilitud (Ross 1987) para ajustar los modelos a los datos obtenidos. La bondad del ajuste fue examinada con un test $\chi^{2}$ (Sokal y Rohlf 1995).

Análisis geoestadístico. En el presente estudio la autocorrelación espacial fue analizada usando variogramas (semivariogramas).

Estimación del semivariograma. El denominado semivariograma experimental se estimó con base en los datos recolectados en los distintos muestreos de la población de muérdago enano en cada parcela experimental. El valor experimental del semivariograma se calculó con la siguiente expresión (Journel y Huijbregts 1978, Isaaks y Srivastava 1989):

$$
\gamma^{*}(h)=\frac{1}{2 N(h)} \sum_{i=1}^{N(h)}\left[z\left(x_{1}+h\right)-z\left(x_{1}\right)\right]^{2}
$$

donde: $\gamma^{*}(h)$ es el valor experimental del semivariograma para el intervalo de distancia $h ; N(h)$ es el número de pares de puntos muestrales separados por el intervalo de distancia $h ; z\left(x_{i}\right)$ es el valor de la variable de interés en el punto muestral $x_{i}$, y $z\left(x_{i}+h\right)$ es el valor de la variable de interés en el punto muestral $x_{i}+h$.
Normalmente el semivariograma es una función monótona no decreciente, ya que al aumentar $h$ también aumenta, al menos en sentido cuadrático, la diferencia entre $Z(x+h)$ y $Z(x)$. Si $Z$ es estacionaria, $\gamma$ alcanza un valor límite constante llamado meseta $(\mathrm{C}+\mathrm{Co})$ que coincide con la varianza $\sigma^{2}$ de $Z$. La distancia a la que se alcanza este valor se denomina rango (a) o alcance y marca la zona de influencia en torno a un punto, más allá de la cual la autocorrelación es nula. Aunque $\gamma(0)=0$, con frecuencia el semivariograma es discontinuo en el origen, con un salto finito que se llama pepita (Co), o efecto pepita (del inglés nugget). El efecto pepita representa la incertidumbre asociada a los datos experimentales o a la variabilidad espacial que pueda existir a una escala de muestreo menor que la efectuada (Samper y Carrera 1996).

Para establecer el semivariograma experimental correspondiente a cada muestreo de las parcelas experimentales se empleó el programa Variowin 2.2 (Pannatier 1996). Se utilizaron semivariogramas omnidireccionales, ya que $a$ priori no había ninguna dirección preferente esperable de máxima variabilidad, por lo que se modelizó la distribución espacial del muérdago enano en cualquier dirección (Isaaks y Srivastava 1989).

Estimación de los parámetros del modelo de semivariograma. Una vez que se calculó el correspondiente semivariograma experimental a cada parcela se le ajustó un semivariograma teórico.

Los modelos teóricos utilizados en este estudio fueron el esférico y el exponencial, siguiendo los parámetros determinados por Samper y Carrera (1996). Para ajustar los correspondientes semivariogramas experimentales a los semivariogramas teóricos de los muestreos en cada parcela experimental se empleó el programa Variowin 2.2. (Pannatier 1996).

Validación. Una vez que los semivariogramas experimentales fueron ajustados a algunos de los modelos teóricos fue necesario validar estadísticamente estos modelos ajustados. La validación de los diferentes modelos teóricos ajustados a los semivariogramas experimentales se realizó con el procedimiento denominado validación cruzada (Isaaks y Srivastava 1989, Hevesi et al. 1992). Los parámetros del modelo a validar $\left(\mathrm{C}_{0}, \mathrm{C}\right.$ y a) se fueron modificando en un procedimiento de prueba y error hasta la obtención de estadísticos de validación cruzada adecuados. Estos estadísticos son los siguientes:

a) Media de los errores de estimación (MEE). Se expresa con la siguiente ecuación:

$$
M E E=\frac{1}{n} \sum_{i=1}^{n}\left[z^{*}\left(x_{i}\right)-z\left(x_{i}\right)\right]
$$


donde: $z^{*}\left(x_{i}\right)$ es el valor estimado de la variable de interés en el punto $x_{i} ; z\left(x_{i}\right)$ es el valor medido de la variable de interés en el punto $x_{i}$, y n es el número de puntos muestrales utilizado en la interpolación. El MEE no debe ser significativamente diferente de 0 (prueba de $\mathrm{t}$ ), en cuyo caso, indicaría que el modelo de semivariograma permite el cálculo de estimaciones no sesgadas.

b) Error cuadrático medio (ECM). Un modelo de semivariograma se considera adecuado si, como regla práctica, el ECM es menor que la varianza de los valores muestrales (Hevesi et al. 1992). La fórmula se expresa de la siguiente manera:

$$
E C M=\frac{1}{n} \sum_{i=1}^{n}\left[z^{*}\left(x_{i}\right)-z\left(x_{i}\right)\right]^{2}
$$

c) Error cuadrático medio adimensional (ECMA). Expresado con la siguiente fórmula:

$$
E C M A=\frac{1}{n} \sum_{i=1}^{n} \frac{\left[z^{*}\left(x_{i}\right)-z\left(x_{i}\right)\right]^{2}}{\sigma k}
$$

donde: $\sigma_{\mathrm{k}}$ es la desviación estándar del error esperado en la estimación con el krigeado. La validez del modelo se satisface si ECMA está comprendido entre los valores $1 \pm 2(2 / \mathrm{N})^{0,5}$.

d) Además se debe cumplir que la varianza de los errores de estimación debe ser $\leq$ que la varianza muestral (Samper y Carrera 1996).

Nivel de dependencia espacial. Para conocer el grado de relación entre los datos correspondientes resulta importante establecer el nivel de dependencia espacial. Este valor se obtuvo al dividir el efecto pepita entre la meseta y expresando en porcentaje el resultado. Si el resultado es menor de $25 \%$ el nivel de dependencia espacial es alto, si se encuentra entre 26 y $75 \%$ el nivel de dependencia espacial es moderado y si es mayor del $76 \%$ el nivel de dependencia es bajo (Cambardella et al. 1994).

Elaboración de mapas. Una vez que los modelos de los semivariogramas correspondientes fueron validados se utilizó el método geoestadístico denominado krigeado para la elaboración de los mapas de densidad de las poblaciones de muérdago enano. El krigeado es un método de interpolación que permite la estima no sesgada de puntos no muestreados. En este trabajo se utilizó el krigeado para obtener la estimación de la densidad del hemiparásito en puntos donde no se contabilizó la presencia del mismo. En este estudio se utilizó el krigeado ordinario para obtener las estimaciones correspondientes. Esta decisión se basó en lo establecido por Samper y Carrera (1996). Las estimaciones de las poblaciones del muérdago enano en cada parcela experimental se realizaron mediante el programa WinGslib 2002.

Una vez obtenidas las estimaciones correspondientes para cada punto no muestreado, se elaboraron los mapas que indicaban la distribución espacial en el campo de las poblaciones del muérdago enano mediante el uso del programa Surfer $9^{1}$. De los mapas elaborados se determinó el porcentaje de superficie infestada por el muérdago enano (presencia-ausencia del hemiparásito).

\section{RESULTADOS}

Resumen estadístico del muérdago enano. El rango de densidad de muérdagos enanos por árbol varió entre 1,47 y 4,28 (cuadro 1). Las parcelas con mayor densidad de muérdagos (3 y 10) se encontraron ubicadas a altitudes más bajas que las otras ocho. Habría que determinar si la altitud en realidad juega algún papel importante para determinar la densidad de este hemiparásito, mediante estudios posteriores. La varianza de los datos se halló entre 5,22 y 53,76 .

Distribución espacial del muérdago enano. El índice de dispersión señala que en todas las parcelas se encontró una distribución agregada del muérdago enano, con excepción de las parcelas 4, 6 y 9, donde indica una distribución aleatoria. Por su parte, el índice de Green confirmó dicha agregación, pero en este caso para todas las parcelas, indicando, en función de su bajo valor (cuadro 2), que la agregación era leve. Estos datos un tanto contradictorios señalan algunas de las limitaciones de estos métodos para determinar con precisión la distribución espacial del hemiparásito.

Por otro lado, con base en las distribuciones estadísticas, los resultados indican que en las parcelas $1,2,4,5,6$, 7, 9 y 10 los datos siguieron una distribución estadística binomial negativa (agregación). En las parcelas 2, 5 y 6 también presentaron un ajuste a la distribución de Poisson (aleatoria), por lo que en estos casos no fue posible discernir la distribución real espacial del muérdago enano, ya que no es posible que el hemiparásito tenga al mismo tiempo dos tipos de distribución espacial. Quizás la explicación a dicha dualidad está en que para valores altos del parámetro $k$ (parámetro asociado a la distribución binomial negativa, que ha sido muy utilizado como otro índice de agregación), como es relativamente el caso, la binomial negativa tiende a la distribución de Poisson (Southwood 1978), por lo que al realizar el ajuste estadístico por máxima verosimilitud se ajusten a los datos ambos modelos indistintamente. Los

\footnotetext{
Surface Mapping System. Golden Software Inc. 2002. 809, 14th Street. Golden, Colorado 80401-1866. USA.
} 
resultados obtenidos por el índice de dispersión y el índice de Green tampoco dejan clara la situación, ya que en los dos primeros casos indican una agregación y en el caso de la parcela 6 el índice de dispersión indica aleatoriedad y el de Green, agregación. Esto demuestra nuevamente las contradicciones en las que pueden caer estos métodos y por lo tanto, señalan sus limitaciones.

Los datos obtenidos en las parcelas 3 y 8 no se pudieron ajustar a ninguna distribución estadística ya que no fue posible obtener la convergencia de los algoritmos de ajuste por máxima verosimilitud.

Análisis geoestadístico. Los modelos de semivariogramas ajustados a los datos del estudio fueron en su mayoría esféricos (cuadro 3). Cabe recordar que los valores presentados son el resultado de la transformación que se realizó.

Con base en la validación cruzada se encontró que en la mayoría de los casos se ajustó un modelo esférico con efecto pepita (cuadro 3), con excepción de las parcelas 2 y 4 , en las cuales se ajustó un modelo exponencial con efecto pepita. Lo anterior permite señalar que las poblaciones de muérdago enano en las 10 parcelas presentaban una distribución espacial agregada.

En el caso del efecto pepita los valores alcanzados fluctuaron entre 0,004 y 0,041. En todos los casos el valor del efecto pepita fue menor del $27 \%$ del valor de la meseta. Lo anterior da pie a deducir que al menos más del $73 \%$ de la variación total era debido a la dependencia espacial encontrada en la escala de muestreo utilizada. Expresado de otra manera, alrededor del $73 \%$ de la variación de la distribución de las poblaciones de muérdago enano se logró explicar por la estructura espacial establecida con los semivariogramas (Liebhold et al. 1993). De acuerdo a lo indicado por Cambardella et al. (1994), el nivel de dependencia espacial de las poblaciones del hemiparásito en todos los casos fue alta, con excepción de la parcela 8 donde fue moderada (cuadro 3).

Con respecto a la meseta, los valores se encontraron entre 0,069 y 0,198 (cuadro 3). En lo referente al alcance los valores se hallaron en el rango entre $43,0 \mathrm{~m}$ y 88,3 m (cuadro 3). Esta distancia indica hasta dónde existía dependencia espacial de los datos muestreados. Más allá de este valor la dependencia espacial era nula. Resulta

Cuadro 1. Resumen estadístico del hemiparásito muérdago enano.

Statistical summary of the parasite dwarf mistletoe.

\begin{tabular}{|c|c|c|c|c|c|}
\hline Parcelas & $\begin{array}{l}\text { Altitud de la parcela } \\
\text { (m s.n.m.) }\end{array}$ & $\begin{array}{c}\bar{x} \\
\text { individuos/parcela }\end{array}$ & S & $\begin{array}{l}\text { Coeficiente de } \\
\text { curtosis }\left(1^{\mathrm{a}}\right)\end{array}$ & $\begin{array}{l}\text { Coeficiente de } \\
\text { curtosis }\left(2^{\mathrm{a}}\right)\end{array}$ \\
\hline $\mathrm{P} 1$ & 3.610 & 3,727 & 3,981 & 4,471 & 1,085 \\
\hline $\mathrm{P} 2$ & 3.696 & 3,381 & 3,440 & 6,983 & $-1,902$ \\
\hline P3 & 3.514 & 4,282 & 2,934 & 10,627 & 0,441 \\
\hline $\mathrm{P} 4$ & 3.721 & 3,300 & 3,333 & 19,552 & 0,833 \\
\hline P5 & 3.802 & 2,300 & 2,395 & 16,561 & $-1,953$ \\
\hline P6 & 3.884 & 1,472 & 2,285 & 6,381 & 1,267 \\
\hline P7 & 3.768 & 1,818 & 3,059 & 10,772 & 0,821 \\
\hline P8 & 3.971 & 2,300 & 3,520 & 5,222 & $-0,661$ \\
\hline P9 & 4.098 & 1,281 & 2,484 & 16,192 & 1,993 \\
\hline P10 & 3.539 & 1,036 & 7,332 & 19,356 & 0,240 \\
\hline
\end{tabular}

Cuadro 2. Índices de dispersión y distribuciones estadísticas del hemiparásito muérdago enano. Indicators of dispersion and distributions statistics parasite dwarf mistletoe.

\begin{tabular}{|c|c|c|c|c|c|}
\hline Parcelas & $\begin{array}{l}\text { Índice de } \\
\text { dispersión }\end{array}$ & Índice de Green & Poisson & $\begin{array}{c}\text { Binomial } \\
\text { negativa }\end{array}$ & $k$ \\
\hline $\mathrm{P} 1$ & $2,32^{\mathrm{s}}$ & 0,002 & NS & $S$ & 1,62 \\
\hline $\mathrm{P} 2$ & $1,56^{\mathrm{s}}$ & 0,003 & $S$ & $S$ & 6,92 \\
\hline P3 & $3,22^{\mathrm{s}}$ & 0,004 & NA & NA & - \\
\hline $\mathrm{P} 4$ & $0,77^{\mathrm{ns}}$ & 0,001 & NS & S & 4,73 \\
\hline P5 & $4,89^{\mathrm{s}}$ & 0,005 & S & $S$ & 9,73 \\
\hline P6 & $0,86^{\mathrm{ns}}$ & 0,001 & $\mathrm{~S}$ & $S$ & 11,35 \\
\hline P7 & $2,71^{\mathrm{s}}$ & 0,003 & NS & $S$ & 5,22 \\
\hline P8 & $3,39^{\mathrm{s}}$ & 0,006 & NA & NA & - \\
\hline P9 & $0,65^{\mathrm{ns}}$ & 0,004 & NS & S & 2,80 \\
\hline P10 & $3,02^{\mathrm{s}}$ & 0,007 & NS & $S$ & 5,65 \\
\hline
\end{tabular}

$\mathrm{S}=$ significativa; $\mathrm{NS}=$ no significativa; nivel de significación al 5\%; NA = No ajustada. 
interesante señalar que la distancia máxima encontrada (88,270 m) es considerable, teniendo en cuenta el tamaño de las parcelas de estudio.

Los valores de los estadísticos de validación cruzada permitieron la validación de los modelos ajustados, al hallarse en los rangos de aceptación matemática (cuadro 4).

Los semivariogramas respectivos (modelos ajustados) elaborados para las poblaciones de muérdago enano en las diferentes parcelas estudiadas se observan en la (figura 1).

Elaboración de mapas de densidad (krigeado). La distribución agregada de las poblaciones del hemiparásito queda de manifiesto en cada uno de ellos (figura 2). El color verde indica la mayor cantidad de muérdagos contabilizados en cada caso; por el contrario, el color blanco indica ausencia de muérdago enano en la zona respectiva. Es importante mencionar que los valores representados son los obtenidos después de la transformación que fue necesario realizar.
En los mapas realizados se aprecian diferentes focos de agregación o de agrupamiento de las poblaciones del hemiparásito repartidos más o menos por todas las parcelas experimentales. Se observa una cierta relación directamente proporcional entre el número de centros de agregación existentes y el promedio de muérdagos detectados en cada caso. El hecho de que fuera posible observar en cada uno de los mapas realizados la presencia de los centros de agregación, confirma lo hallado con la geoestadística.

Superficie infestada. De manera general, en los mapas establecidos con el krigeado se observó que la superficie libre de infestación disminuyó conforme la densidad media era mayor (cuadro 5). De esta manera se detectó que en las parcelas 1 y 3 que presentaron una alta densidad del hemiparásito, la superficie sin infestación fue baja (8,9 y $5,2 \%$, respectivamente) y, por el contrario, en las parcelas 9 y 10 con menor densidad de muérdago enano, la superficie no infestada fue mayor (30,2 y 30,4\%, respectivamente).

Cuadro 3. Parámetros (efecto pepita, meseta y alcance) de los modelos ajustados a los semivariogramas del número de muérdagos enanos.

Parameters (Effect Pepita, Plateau and Scope) of the models adjusted to semivariograms the number of dwarf mistletoe.

\begin{tabular}{|c|c|c|c|c|c|c|}
\hline Parcelas & Modelo & Pepita & Meseta & $\begin{array}{l}\text { Alcance } \\
\text { (metros) }\end{array}$ & $\begin{array}{c}\text { Pepita/ } \\
\text { Meseta }(\%)\end{array}$ & $\begin{array}{c}\text { Nivel de } \\
\text { dependencia espacial }\end{array}$ \\
\hline P1 & Esférico & 0,020 & 0,156 & 57,3 & 12,8 & Alta \\
\hline $\mathrm{P} 2$ & Exponencial & 0,004 & 0,144 & 83,5 & 2,8 & Alta \\
\hline $\mathrm{P} 3$ & Esférico & 0,017 & 0,069 & 43,1 & 24,6 & Alta \\
\hline P4 & Exponencial & 0,006 & 0,138 & 88,3 & 4,3 & Alta \\
\hline P5 & Esférico & 0,010 & 0,108 & 53,2 & 9,3 & Alta \\
\hline P6 & Esférico & 0,018 & 0,111 & 46,8 & 16,2 & Alta \\
\hline P7 & Esférico & 0,030 & 0,120 & 51,3 & 25,0 & Alta \\
\hline P8 & Esférico & 0,034 & 0,130 & 52,4 & 26,2 & Moderada \\
\hline P9 & Esférico & 0,041 & 0,190 & 59,8 & 21,6 & Alta \\
\hline P10 & Esférico & 0,031 & 0,198 & 52,9 & 15,7 & Alta \\
\hline
\end{tabular}

Cuadro 4. Valores de los estadísticos de la validación cruzada: media de los errores de estimación (MEE), error cuadrático medio (ECM) y error cuadrático medio adimensional (ECMA), del muérdago enano.

Values of statistical validation crusade: average errors estimate (MEE), mean squared error (ECM) and mean squared error adimensional (ECMA), the dwarf mistletoe.

\begin{tabular}{|c|c|c|c|c|c|c|}
\hline Parcela & $\begin{array}{c}\text { Media } \\
\text { muestral }\end{array}$ & $\begin{array}{l}\text { Varianza } \\
\text { muestral }\end{array}$ & MEE & $\begin{array}{c}\text { Varianza } \\
\text { de los errores }\end{array}$ & ECM & ECMA \\
\hline P1 & 0,505 & 0,164 & $0,10 \mathrm{~ns}$ & 0,105 & 0,122 & 1,03 \\
\hline $\mathrm{P} 2$ & 0,489 & 0,151 & $0,11 \mathrm{~ns}$ & 0,118 & 0,140 & 1,10 \\
\hline P3 & 0,649 & 0,073 & $0,10 \mathrm{~ns}$ & 0,021 & 0,051 & 1,01 \\
\hline $\mathrm{P} 4$ & 0,478 & 0,150 & $0,10 \mathrm{~ns}$ & 0,123 & 0,133 & 1,04 \\
\hline P5 & 0,398 & 0,112 & $0,12 \mathrm{~ns}$ & 0,091 & 0,095 & 1,11 \\
\hline P6 & 0,249 & 0,110 & $0,09 \mathrm{~ns}$ & 0,085 & 0,088 & 1,03 \\
\hline P7 & 0,291 & 0,118 & $0,15 \mathrm{~ns}$ & 0,097 & 0,100 & 1,12 \\
\hline P8 & 0,333 & 0,146 & $0,07 \mathrm{~ns}$ & 0,117 & 0,122 & 1,08 \\
\hline P9 & 0,342 & 0,209 & $0,08 \mathrm{~ns}$ & 0,181 & 0,194 & 1,10 \\
\hline P10 & 0,426 & 0,218 & $0,11 \mathrm{~ns}$ & 0,190 & 0,201 & 1,07 \\
\hline
\end{tabular}

* Tamaño de muestra $=121$ en cada parcela. ns: diferencia no significativa al $5 \%$. 

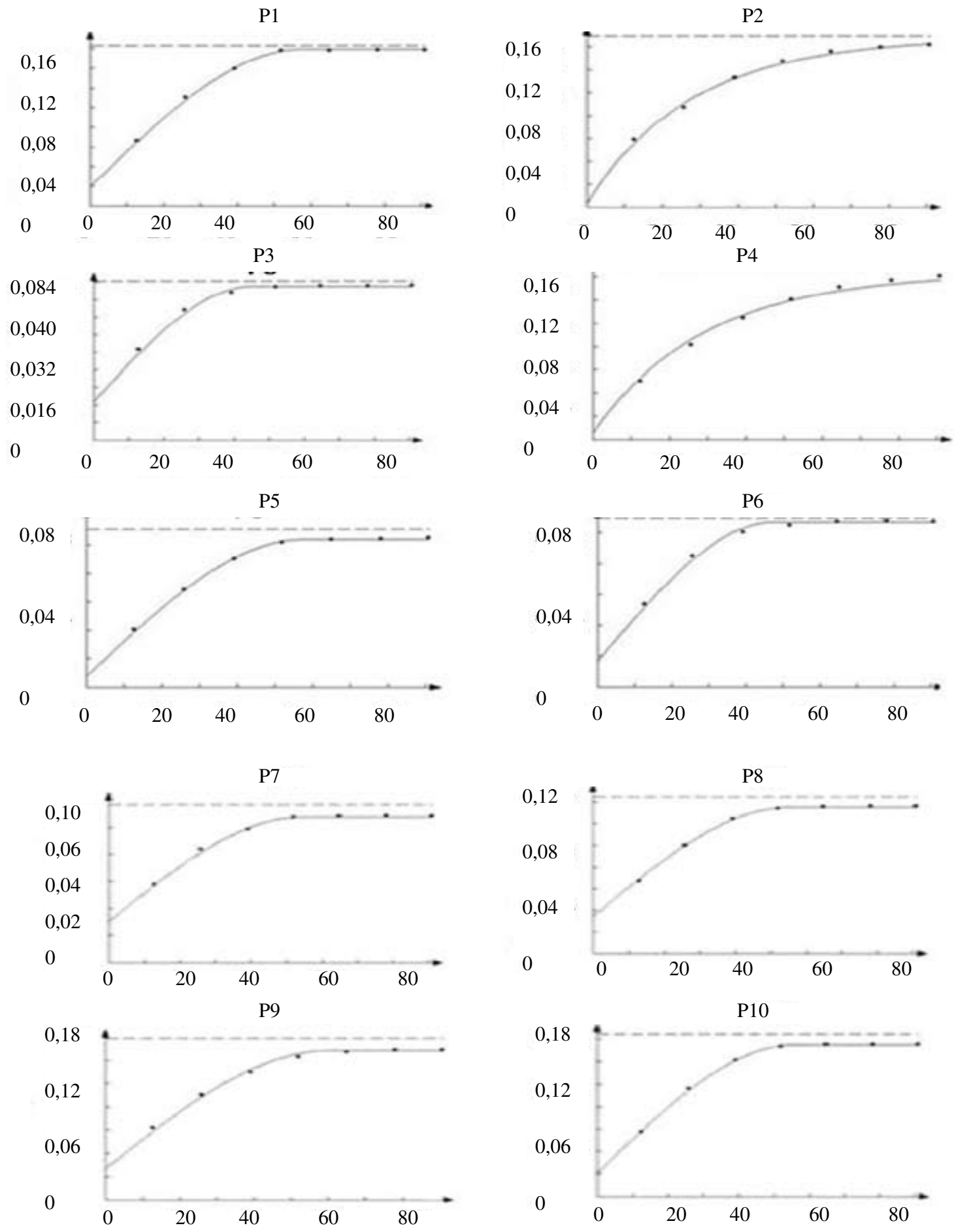

Figura 1. Semivariogramas de la distribución espacial de muérdago enano.

Semivariograms of the spatial distribution of the dwarf mistletoe. 

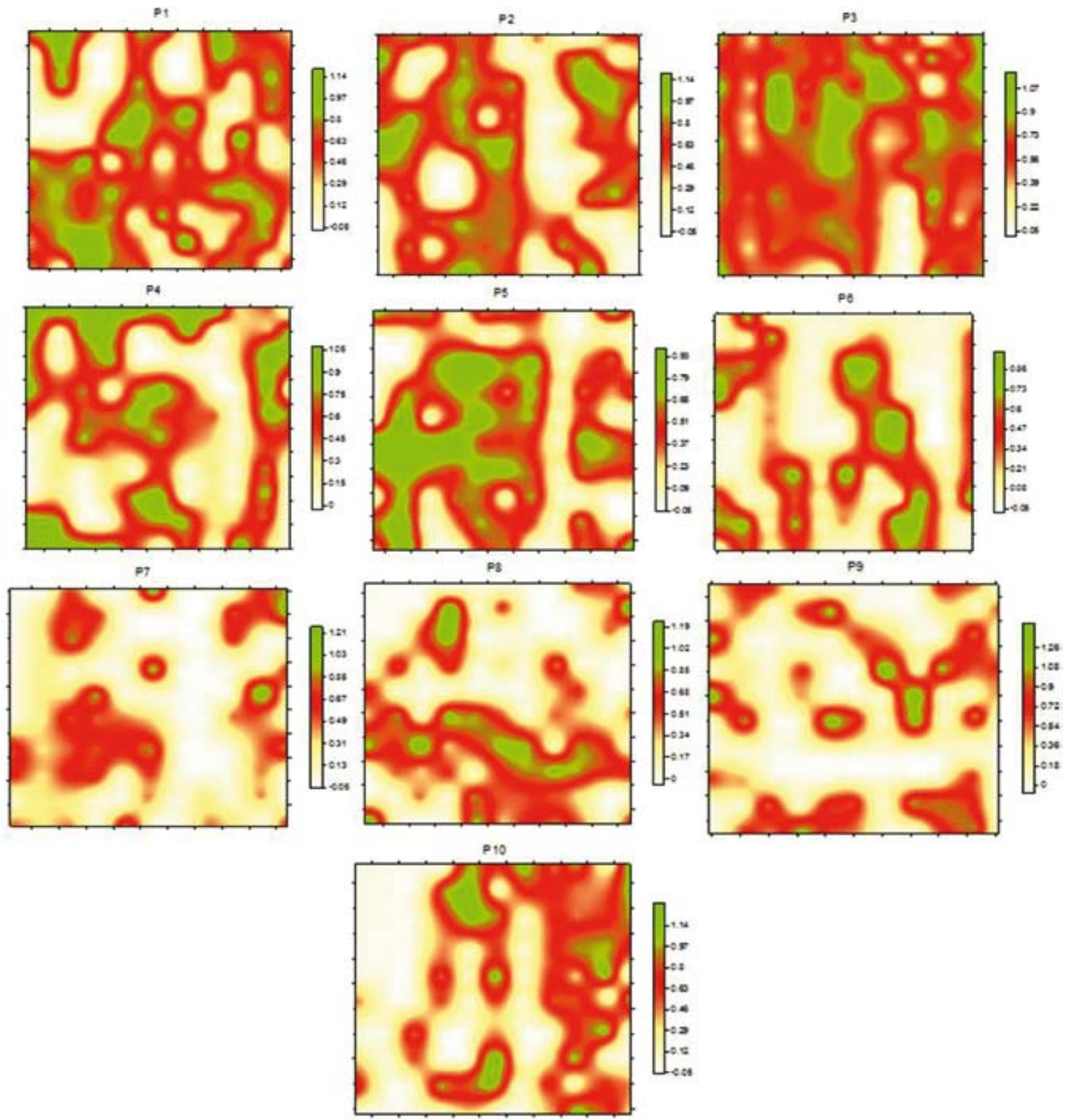

Figura 2. Mapas de distribución del muérdago enano desarrollados mediante krigerado para cada parcela experimental. Density population maps of dwarf mistletoe developed with Krige, for each of the experimental plots. 
Cuadro 5. Porcentaje de superficie infestada y no infestada por el muérdago enano.

Percentage of infested area and not infested by dwarf mistletoe.

\begin{tabular}{lccc}
\hline & $\bar{x}$ & \multicolumn{2}{c}{ Krigeado (mapas) } \\
\cline { 3 - 4 } Parcelas & $\begin{array}{c}\% \\
\text { individuos/ } \\
\text { parcela }\end{array}$ & $\begin{array}{c}\% \\
\text { Infestado }\end{array}$ & $\begin{array}{c}\% \\
\text { No infestado }\end{array}$ \\
\hline P1 & 3,727 & 85,4 & 8,9 \\
P2 & 3,381 & 80,6 & 19,4 \\
P3 & 4,282 & 94,8 & 5,2 \\
P4 & 3,300 & 88,7 & 11,3 \\
P5 & 2,300 & 91,1 & 14,6 \\
P6 & 1,472 & 77,7 & 22,3 \\
P7 & 1,818 & 71,3 & 28,7 \\
P8 & 2,300 & 74,2 & 25,8 \\
P9 & 1,281 & 69,8 & 30,2 \\
P10 & 1,036 & 69,6 & 30,4 \\
\hline
\end{tabular}

\section{DISCUSIÓN}

Los índices de dispersión utilizados en este trabajo muestran un escenario general de agregación en el comportamiento espacial del muérdago enano. Aunque el índice de dispersión (cociente media-varianza) y el índice de Green indican de manera general resultados similares en cuanto a la detección del agregamiento, el grado de agregación fue diferente. En general el índice de Green determina un mayor número de agregaciones leves. Si bien este índice es presentado como el más adecuado de los índices de dispersión ya que es independiente del número total de individuos capturados y del valor medio de su abundancia, no es independiente del tamaño de la muestra (De los Santos et al. 1982). Por su parte, el índice de dispersión (cociente varianza-media) al utilizar la varianza para evaluar el grado de agregación presenta graves inconvenientes ya que depende directamente de la abundancia (Leveche 1972), lo que hace que poblaciones con densidades medias altas presenten valores altos del índice limitando en gran medida la fiabilidad del resultado obtenido.

El uso de distribuciones estadísticas señala resultados similares a los de los índices de dispersión, con un ajuste casi general a la distribución binomial negativa. Dicho modelo tiene una larga tradición en la literatura ecológica para explicar el comportamiento espacial de los organismos (Pielov 1977). El valor $k$ de la distribución binomial negativa es en sí mismo un índice de contagio y está relacionado inversamente con el patrón de agregación (Southwood 1978, Taylor 1984).

En los resultados de este estudio dicho valor oscila entre 1,62 y 11,35. Los valores más bajos y, por lo tanto, donde la agregación es más fuerte, según los autores citados, se hallan en las parcelas 1 y 9 . Este índice, sin embargo, está influenciado por el tamaño de la muestra y la unidad de muestreo. Si se logran mantener constantes estos factores, este índice proporciona una media muy útil del nivel de agregación de una población, siendo a la vez muy sensible al tipo de hábitat y al estadío de desarrollo de las poblaciones (Hairston 1959). En este estudio el tamaño de la muestra presenta variabilidad, ya que la densidad del muérdago enano en cada parcela fue diferente, por lo que pudo afectar la efectividad de este valor $k$.

Como se aprecia, los métodos estadísticos no espaciales utilizados para establecer la distribución espacial de las poblaciones del muérdago enano tienen deficiencias y limitaciones. En muchas ocasiones los resultados parecen ser contradictorios, en otros casos no se ajustan los modelos de distribuciones espaciales y en otras circunstancias se ajustan los datos a los dos modelos de distribución. A ello hay que añadir otra gran limitación de estos métodos como es que no tienen en cuenta la naturaleza bidimensional de las poblaciones, es decir, la localización exacta de los individuos muestreados. Por consiguiente, no utilizan toda la información espacial disponible, lo que limita su alcance.

La distribución espacial agregada del muérdago enano fue reportada por Gutiérrez (1970) y Andrade (1981), en ambos casos los autores utilizaron distribuciones espaciales en sus estudios y reportaron algunos problemas con el ajuste estadístico correspondiente, como sucedió en este estudio. Rodríguez (1985) y Hernández (1994) determinaron el mismo comportamiento espacial del hemiparásito utilizando diversos índices de dispersión y reportaron asimismo, problemas en la determinación de la distribución espacial del muérdago enano.

Dentro del área de la estadística espacial es cada vez más común el uso de la geoestadística como una herramienta de modelización de multitud de fenómenos biológicos (Rossi et al. 1992). La aplicación de la geoestadística a los datos de este estudio permite modelizar la estructura espacial del hemiparásito de coníferas con modelos esféricos con efecto pepita en la mayoría de los casos y exponencial con efecto pepita, en algunos casos. Los resultados obtenidos determinan la existencia de una estructura espacial agregada, siendo el nivel de dependencia de la misma, en general, alto. Ello indica que el efecto pepita no fue demasiado acusado, lo anterior, aunado a la pequeña varianza de los datos, permite afirmar que la escala de estudio fue la adecuada y el error de muestreo no fue demasiado importante (Oliver y Webster 1991, Rossi et al. 1992, McBratney y Pringle 1999). Resultaría interesante, en estudios posteriores, determinar si la escala de muestreo o el tamaño de las parcelas de estudio pudieran tener un efecto sobre el nivel de dependencia espacial detectado en las poblaciones del hemiparásito.

Hay que destacar el hecho de que las diferentes altitudes en las cuales fueron establecidas las parcelas de estudio y el diámetro del tronco del pino, al parecer no influyen en el establecimiento de la agregación por parte de las poblaciones de muérdago enano, tal y como se aprecia en los mapas. 
Aunque no se determinó estadísticamente, se pudo observar en campo que, tomando en cuenta que el diámetro del tronco de los pinos está en relación con su edad, el hemiparásito no parece tener preferencias de infestación en cuanto a la edad y talla del pino. Lo anterior resulta interesante ya que Gutiérrez (1970) señala que la infestación del muérdago enano influye sobre el desarrollo en grosor del fuste del pino, por lo tanto, el ataque a pinos jóvenes reduciría el desarrollo posterior de estos árboles. Tomando en cuenta que una parte considerable de pinos muestreados en este estudio era relativamente joven, el impacto sobre su desarrollo tendrá gran importancia dentro del contexto ambiental del PNNT. La anterior aseveración habría que corroborarla estableciendo un estudio en el que se demuestre estadísticamente que el hemiparásito infesta de forma generalizada sin importar edad y talla del pino.

Pese a que el muérdago enano se encuentra en condiciones óptimas para su desarrollo, la superficie infestada por parcela, en porcentaje, nunca llegó al $100 \%$. Por lo tanto, las acciones de control podrían ser dirigidas sobre zonas específicas de infestación y no sobre la suposición de una colonización del $100 \%$ por parte del hemiparásito, tal y como proponía en su momento González (1984) con la utilización de herbicidas selectivos.

Es importante resaltar que, contando con las áreas específicas de infestación de este hemiparásito sobre la zona de estudio, se podrían establecer programas de manejo de este problema sanitario del PNNT en su ladera norte utilizando técnicas de precisión, como por ejemplo la adecuación de las propuestas sugeridas por Fleischer et al. (1999). Con lo anterior, se podrían reducir los costos económicos y medioambientales, al reducir el uso de herbicidas selectivos en el control de esta plaga.

Como se mencionó anteriormente, la distribución agregada del hemiparásito ya fue reportada por Gutiérrez (1970), Andrade (1981), Rodríguez (1985) y Hernández (1994). Sin embargo, estos autores reportaron problemas con los métodos de estadística no espacial. En este estudio, el análisis geoestadístico permitió, sin lugar a dudas, determinar el comportamiento agregado de las poblaciones del muérdago enano, a través de los modelos de semivariogramas, la estimación del krigeado ordinario y los mapas elaborados para cada parcela de estudio.

\section{CONCLUSIONES}

Los métodos no espaciales (distribuciones estadísticas e índices de dispersión) muestran de manera general un comportamiento agregado de las poblaciones del muérdago enano. Sin embargo, presentan serias deficiencias y limitaciones.

La utilización de la geoestadística permite modelizar la estructura espacial de las poblaciones del muérdago enano con modelos esféricos y exponenciales con efecto pepita. Los resultados obtenidos con las técnicas geoestadísticas indican la existencia de una estructura espacial agregada, siendo alto el nivel de dependencia espacial.

La altitud sobre el nivel del mar y el diámetro del tronco del pino no influyen en el establecimiento de la agregación por parte de las poblaciones del muérdago enano.

Los mapas geoestadísticos obtenidos muestran ser adecuados y eficientes para el estudio del comportamiento espacial del hemiparásito.

Existen áreas libres de infestación que permitirían una aplicación dirigida de las medidas de control establecidas para este hemiparásito y la reducción de los costos económicos y medioambientales.

\section{REFERENCIAS}

Andrade EV. 1981. Evaluación de Efectos del Muérdago Enano (Arcetobium globosum Hawk Wiens, y A. vaginatum Wild) en rodales de Pinus hartwegii Lind. Ciencia Forestal 45(3): 10-17.

Blom E, S Fleischer. 2001. Dynamics in the spatial structure of Leptinotarsa decemlineata (Coleoptera: Chrysomelidae). Environ. Entomol. 30(2): 350-364.

Cambardella C, T Moorman, J Novak, T Parkin, D Karlen, R Turco, A Konopka. 1994. Field scale variability of soil properties in central iwa soils. Soil. Sci. Soc. Am. J. 58: 1501-1511.

CEPANAF (Comisión Estatal de Parques Nacionales Federales, MX). 1979. Programa de manejo del Parque Nacional Nevado de Toluca. 106 p. (Informe de Acciones № 22).

Chauvet P. 1968. Aide-Memoire de Geostatistique Miniere. Paris, Francia. École des Mines. 210 p.

Chilés JP, P Delfiner. 1999. Geostatistics Modelling Spatial Uncertainty. New York, USA. John Wiley. 695 p.

De los Santos A, C Montes, L Ramírez. 1982. Modelos espaciales de algunas poblaciones de coleópteros terrestres en dos ecosistemas del bajo Guadalquivir (S.W. España). Mediterránea Ser. Biol. 6: 65-92.

Fleischer J, E Blom, R Weisz. 1999. Sampling in Precision IPM : When The Objetive Is a Map. The American Phytopathological Society 89(11): 115-118.

García E. 1976. Modificaciones al sistema de clasificación climática de Köppen. México DF, México. Talleres de offset Larios. 365 p.

González CE. 1984. Problemas de Plagas y Enfermedades del Pinus radiata. D. Don., en la Ciudad de México y Áreas colindantes. Ciencia Forestal 49 (9): 15-22.

Gutiérrez R. 1970. Efectos del parasitismo del muérdago enano (Arceothobium $\mathrm{sp}$ ) sobre el desarrollo en grosor del fuste de Pinus moctezumae Lamb. y Pinus hartwegii Lind en el cerro de Telaron, Estado de México, México. INIF. 22 p. (SARH Boletín Técnico. No. 34).

Hairston NG. 1959. Species abundance and organisation. Ecology. 40: 404-416.

Hernández V. 1994. Evaluación de la infección causada por los muérdagos Struthanthus deppeanus (cham \& schlecht.) y Arceuthobium gilli Subs. NigrumHawks \& Wiens en Pino de la Sierra Negra de Puebla. Tesis Ingeniero Agrónomo. Texcoco, México. Facultad de Agronomía. Universidad Autónoma de Chapingo. 93 p. 
Hevesi J, J Istok, A Flint. 1992. Precipitation estimation in mountainous terrain using multivariate geostatistics. Part. I. Structural analysis. Journal of applied meteorology 31(7): 661-676.

Hulbert S. 1990. Spatial distribution of the montane unicorn. Oikos 58: 257-71.

Isaaks E, RM Srivastava.1988. Spatial continuity measures for probabilistic and deterministic geostatistics. Mathematical Geology 20(4): 313-341.

Isaaks E, RM Srivastava. 1989. An Introduction to Applied Geostatistics. New York, USA. Oxford University Press. $561 \mathrm{p}$.

Journel AG, CJ Huijbregts. 1978. Mining Geostatistics. Londres, Reino Unido. Academic Press. 600 p.

Leveche Ch. 1972. Mollusques benthiques du lac Tchad: ecologie, etude des peuplements et estimation des biomasses. Cah. O. R. S. T. O. M., ser Hidrobiol. 6(1): 3-45.

Liebhold A, E Rossi, P Kemp. 1993. Geostatistics and Geographic Information Systems in Applied Insect Ecology. Annu. Rev. Entomol. 38: 303-327.

Matheron G. 1971. The theory of regionalized variables and its applications. Paris, Francia. Les Cahiers du CMM, Fasc. $\mathrm{N}^{\circ}$ 5, ENSMP. $211 \mathrm{p}$.

McBratney A, M Pringle.1999. Estimating average and proportional variograms of soil properties and their potential use in precision agriculture. Precision Agriculture 1: 125-152.

Oliver M, R Webster. 1991. How geostatistics can help you. Soil Use and Management 7(4): 206-217.

Pannatier Y. 1996. VARIOWIN: Software for Spatial Data Analysis in 2D., New York, USA. Springer Verlag. 91 p.

Pielov E. 1977. Mathematical Ecology. New York, USA. John Wiley. 386 p.

PROTIMBOS (Protectora e Industrializadora de Bosques, MX). 1972. Síntesis del Proyecto Nevado de Toluca; Reforestación a 8 años. 74 p. (Boletín Forestal № 11).
Rodríguez. A. 1985. Infestación de muérdago enano Arcetobium vaginatum (Willd), en el repoblado de Pinus hartwegii, en el Parque Nacional Zoquiapan, Estado de México, México. 165 p. (Boletín Técnico No 122 SARH).

Ross CJ. 1987. Maximum likelihood program. Londres, Reino Unido. Rothamsted Experimental Station Harpenden. $295 \mathrm{p}$.

Rossi R, J Mulla, G Journel, H Franz. 1992. Geostatical tools for modeling and interpreting ecological spatial dependence. Ecological Monographs. 62(2): 277-314.

Samper FJ, J Carrera. 1996. Geoestadística: aplicaciones a la hidrología subterránea. Barcelona, España. Centro Internacional de Métodos en Ingeniería. 484 p.

Sawyer J. 1989. Inconstancy of taylor's b: simulated sampling with different quadrat sizes and spatial distributions. Res. Popul. Ecol. 31: 11-24.

Secretaría de Ecología. 1999. Programa de Manejo del Parque Nacional Nevado de Toluca. Toluca, Estado de México. Editado por el Gobierno del Estado de México. 213 p.

Sokal R, F Rohlf. 1995. Biometry: The principles and practice of statistics in biological research. New York, USA. John Wiley. $356 \mathrm{p}$.

Southwood TR. 1978. Ecological Methods. New York, USA. Wiley/Halsted. $524 \mathrm{p}$.

Taylor L. 1984. Assesing and interpreting the spatial distributions of insect populations. Annu. Rev. Entomol. 29: 321-357.

Vega R. 1976. El muérdago enano: enfermedad creciente en bosques de coníferas en México. México. Departamento de Divulgación Forestal. S.E.R.A. 50 p.

Verduzco J. 1976. Protección forestal. Texcoco, México. Patena (Chapingo).120 p.
Recibido: 21.07 .08

Aceptado: 07.09.09 